\title{
HD 199143 and HD 358623: Two recently identified members of the $\beta$ Pictoris moving group
}

\author{
D. Kaisler ${ }^{1}$, B. Zuckerman ${ }^{1}$, I. Song ${ }^{1,2}$, B. A. Macintosh ${ }^{3}$, A. J. Weinberger ${ }^{4}$, \\ E. E. Becklin ${ }^{1}$, Q. M. Konopacky ${ }^{1,3}$, and J. Patience ${ }^{3,5}$ \\ 3 Institute for Geophysics and Planetary Physics, Lawrence Livermore National Labs, USA \\ ${ }^{4}$ Department of Terrestrial Magnetism, Carnegie Institution of Washington, USA \\ 5 Division of Physics, Mathematics, and Astronomy, California Institute of Technology, USA
}

1 Department of Physics and Astronomy, UC Los Angeles, USA

2 Institute for Geophysics and Planetary Physics, UC Los Angeles, USA

Received 18 March 2003 / Accepted 9 October 2003

\begin{abstract}
HD 199143 and HD 358623 (BD-17 6128 ) are two sets of binary stars which are physically associated and 48 pc from Earth. We present heliocentric radial velocities and high lithium abundances which establish these stars as members of the $\sim 12$ Myr-old $\beta$ Pictoris Moving Group. We also present mid-IR photometric measurements which show no firm evidence for warm dust around all four stars.
\end{abstract}

Key words. stars: individual: HD 199143, HD 358623 - stars: circumstellar matter open clusters and associations: individual: $\beta$ Pictoris

\section{Introduction}

The discovery of an increasingly large number of young stars near Earth is providing fertile ground for studies of Galactic star formation, including the initial kinematics of the local stellar population, the evolution of the stars themselves, and the formation of their primordial disks. During the past two years, a number of authors have presented data on the rapidly rotating F8V star HD 199143 and the K7-M0 dwarf HD 358623. Based on their common proper motion, van den Ancker et al. (2000) (hereafter vdA00) concluded that these stars are associated and have an age of $20 \mathrm{Myr}$. To explain EUVE and ROSAT (Voges et al. 1999, 2000) detections, rapid rotation, variability in the continuum and emission lines of HD 199143, vdA00 postulated a protoplanetary disk or unseen stellar companion. Abundant lithium $(E W=400 \mathrm{~m} \AA$; Mathioudakis et al. 1995) in HD 358623 and chromospheric activity of both stars also indicate young ages. van der Ancker et al. (van den Ancker et al. 2001, vdA01) drew on the similar proper motions of these two stars to classify them as a new young cluster, which they named the Capricornus association.

Adaptive optics (AO) imaging by Jayawardhana \& Brandeker (2001 hereafter, JB01) revealed companion candidates to HD 199143 and HD 358623 at separations of 1 and $2^{\prime \prime}$ respectively. Astrometry by Neuhäuser et al. (2002) firmly established HD $358623 \mathrm{~B}$ as a true companion of

Send offprint requests to: D. Kaisler, e-mail: Kaisler@astro.ucla.edu
HD 358623 A. vdA01 measured $N(11 \mu \mathrm{m})$ and $Q(19 \mu \mathrm{m})$ band excesses around both HD 199143 and HD 358623 implying circumstellar disks. Near-IR photometry by JB01 resulted in an extremely red color $(J-K=1.4)$ for HD 199143 B which was interpreted as evidence of a circumsecondary disk. However, Chauvin et al. (2002) later showed that this photometry was highly dependent upon the deconvolution parameters and suggested that this influenced the assignment of an infrared excess to HD 199143.

Despite these discoveries, the reported ages of these stars have been inconsistent. Since age is the key factor in constraining models of lithium depletion, dust disk dissipation, and planetary formation, it is desirable to have an accurate estimate of the ages of these stars. In this paper, we present near- and midinfrared photometry as well as optical spectroscopy to show that the space motions and spectral features of HD 199143 and HD 358623 are consistent with those of the $\beta$ Pictoris Moving Group; from this, and from high lithium abundances, we infer young ages for the two systems.

\section{Observations}

\subsection{Near-IR photometry with adaptive optics}

We observed HD 199143 and HD 358623 at four epochs (Table 1). The AO system at the Shane 3-m telescope at Lick observatory was used with IRCAL, a $256 \times 256$ Rockwell $\mathrm{HgCdTe}$ array with a plate scale of $0.0756 \mathrm{arcsec} / \mathrm{pixel}$ (Lloyd et al. 2000). Strehl ratios (at $2.1 \mu \mathrm{m}$ ) were in the range 
Table 1. Near-infrared observing Log.

\begin{tabular}{llll}
\hline \hline Target & Epoch \& UT & Filter & Instrument \\
\hline HD 199143 & $2001-06-14$ & $K_{\mathrm{s}}$ & IRCAL \\
& $2001-06-14$ & $J$ & “ \\
& $2001-07-03$ & $K^{\prime}$ & SCAM \\
& $2001-09-29$ & $K_{\mathrm{s}}$ & IRCAL \\
& $2001-09-30$ & $J$ & IRCAL \\
& $2002-06-30$ & $K_{\mathrm{s}}$ & IRCAL \\
& $2002-06-30$ & $J$ & “ \\
HD 358623 & $2001-06-14$ & $K_{\mathrm{s}}$ & IRCAL \\
& $2001-06-14$ & $J$ & “ \\
& $2001-07-03$ & $K^{\prime}$ & SCAM \\
& $2002-06-30$ & $K_{\mathrm{S}}$ & IRCAL \\
& $2002-06-30$ & $J$ & “ \\
\hline
\end{tabular}

Table 2. Near-IR photometry

\begin{tabular}{llll}
\hline \hline Star & $J$ & $K$ & $J-K$ \\
\hline HD 199143 A & $6.24 \pm 0.03$ & $5.89 \pm 0.04$ & $0.35 \pm 0.05$ \\
HD 199143 B & $8.92 \pm 0.07$ & $8.01 \pm 0.07$ & $0.91 \pm 0.10$ \\
HD 358623 A & $7.92 \pm 0.03$ & $7.12 \pm 0.04$ & $0.80 \pm 0.05$ \\
HD 358623 B & $9.67 \pm 0.05$ & $8.77 \pm 0.06$ & $0.90 \pm 0.08$ \\
\hline
\end{tabular}

0.3-0.45. Absolute photometry for HD 199143 and HD 358623 was obtained on 30 June 2002 by comparing open-loop images with the standard star HD 201941 (Elias et al. 1982). Measurements were done with IRAF using an aperture radius of 40 pixels ( $\left.3^{\prime \prime}\right)$. Although the $K^{\prime}$ and $K_{\mathrm{s}}$ filters used for this study have slightly shorter bandpasses than the Elias et al. (1982) $K$-band filter, Persson et al. (1998) find that the color-correction between $K$ and $K_{\mathrm{s}}$ is $<0.02$ mag even for stars as red as HD 199143 and HD 358623. Therefore, we have made no color correction here, but have added in quadrature an additional $0.02 \mathrm{mag}$ error to the quoted $K$ magnitude (Table 2).

At the Keck II telescope, we used the AO system with NIRSPEC's slit-viewing camera, SCAM (McLean et al. 1998). The plate scale for SCAM's $256 \times 256 \mathrm{HgCdTe}$ array is $0 .{ }^{\prime} 0167 \pm 0 . ' 001 /$ pixel. Strehl ratios for this run $\left(K^{\prime}\right.$-band $)$ were $0.10-0.46$.

Photometric measurements of HD 199143 AB were performed using the Starfinder routine (Diolaiti et al. 2000) and are reported in Table 2. Our estimates of the magnitudes of these stars agree well with those of other studies except in the case of the JB01 J-band measurement of HD 199143 B. However, Chauvin et al. (2002) note that the JB01 value may be brought into agreement with the others by the choice of a more robust myopic deconvolution algorithm.

On 14 June 2001, we measured the binary separations and position angles as 1'.02 \pm 0. '03 and $-36.0 \pm 0.5$ for HD 199143 and $2^{\prime \prime} .12 \pm 0.02$ and $137^{\circ} .9 \pm 0.5$ for HD 358623. Our position angles differ slightly from those published by JB01, however due to the superior weather conditions under which our observations were made (Chauvin et al. 2002), we believe that our measurements are more accurate.

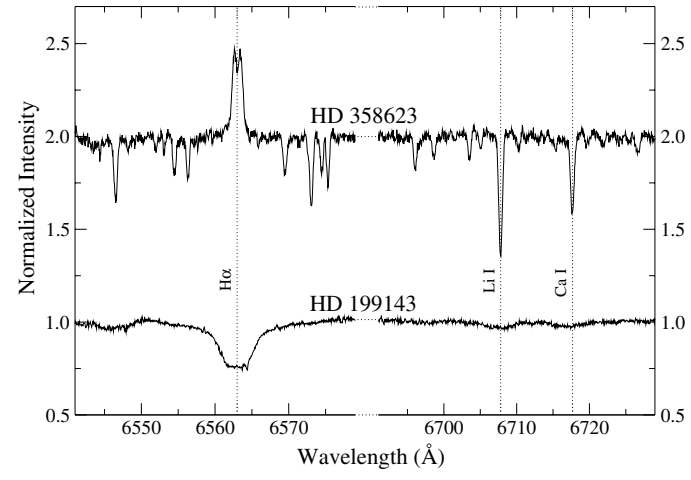

Fig. 1. Hamilton Echelle spectra of HD 199143 and HD 358623.

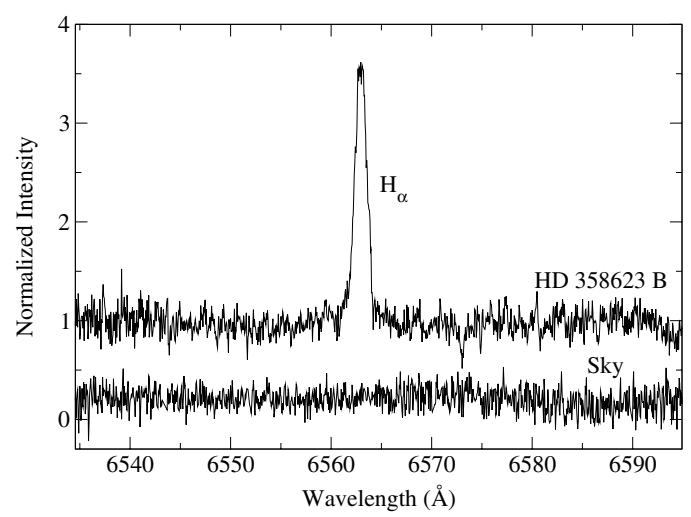

Fig. 2. Hamilton spectra of HD 358623 B. To estimate the contamination from the primary, we took an empty sky spectrum with the same separation from the primary and opposite position angle. Equivalent width of the $\mathrm{H} \alpha$ line is $\sim 4 \AA$.

\subsection{Optical spectroscopy}

We obtained high-resolution $(\sim 0.15 \AA)$ spectra of HD 199143 and HD 358623 (Fig. 1) with the Hamilton Echelle spectrometer (Vogt 1987) at the Shane Telescope. HD 358623 was observed on UT 18 June 2001 and both HD stars were observed on 29 July 2001 . Seeing was $\sim 1^{\prime \prime}$ on both nights. Spectra were reduced using IRAF following a standard procedure (flat-field correction, etc.). Equivalent widths of $\mathrm{H} \alpha$ and Li 6708 features were measured using the IRAF task, splot. Terrestrial atmospheric features were removed using an A-type rapid rotator, HR 7235.

The heliocentric radial velocity of HD 358623 (Table 3) was measured by cross-correlating its spectrum with radial velocity standards HR 911, HR 6349, HR 8232, and HR 8969. All standards gave consistent results. The weighted mean of the measured radial velocities of HD 358623 is $-6.8 \pm 1.3 \mathrm{~km} \mathrm{~s}^{-1}$.

To verify that our $\mathrm{H} \alpha$ spectrum of HD 358623 B was uncontaminated by light from HD 358623, we placed the slit first on $\mathrm{B}$ and then on a blank field at equal distance from and opposite position angle with respect to A. Figure 2 confirms that there is no scattered light from the primary star at this distance. The projected dimensions of the slit used for this observation 
Table 3. Results from optical spectroscopy.

\begin{tabular}{lllll}
\hline \hline Target & $\begin{array}{l}E W(\mathrm{H} \alpha) \\
{[\AA]}\end{array}$ & $\begin{array}{l}E W(\mathrm{Li}) \\
{[\mathrm{m} \AA]}\end{array}$ & $\begin{array}{l}v \sin i \\
{\left[\mathrm{~km} \mathrm{~s}^{-1}\right]}\end{array}$ & $\begin{array}{l}v_{r} \\
{\left[\mathrm{~km} \mathrm{~s}^{-1}\right]}\end{array}$ \\
\hline HD 199143 A & 1.6 & $120:$ & $120^{a}$ & - \\
HD 358623 A & $-0.78^{b}$ & $400 \pm 20$ & 13 & $-6.8 \pm 1.3$ \\
HD 358623 B & $\sim-4^{b}$ & - & - & - \\
\hline
\end{tabular}

${ }^{a}$ Uncertainty $\sim 10-20 \%$.

${ }^{b}$ Emission line.

are 1.5 arcsec wide in the dispersion direction and 2.5 arcsec long in the spatial direction.

\subsection{Mid-infrared photometry}

Mid-infrared observations of HD 199143 and HD 368623 were taken on 7 Dec. and 21 Dec. 2001 at the W. M. Keck Observatory with the facility Long Wavelength Spectrograph (LWS) (Jones \& Puetter 1993). LWS's $128 \times 128$ pixel array has a plate scale of $0.08 \mathrm{arcsec}^{\mathrm{axel}}{ }^{-1}$ and a focal-plane field of view of $10 ! 2$ square. The conditions were photometric during both sets of observations. The image quality was limited by seeing to FWHM of 0.5 at $12 \mu \mathrm{m}$ and 0.6 at $18 \mu \mathrm{m}$ on 7 Dec. and 0.8 at $8-12 \mu \mathrm{m}$ and 0.6 at $18 \mu \mathrm{m}$ on 21 Dec.

The images were taken in a chop-nod mode with a chop amplitude of $10^{\prime \prime}$, a nod of $10^{\prime \prime}$ in the same direction, and a chopper frequency of $5 \mathrm{~Hz}$. This scheme produces four images, two of which contain the object and two of which contain background only. The four images were double differenced for optimal background subtraction of the sky and telescope thermal emission. Filter characteristics are tied to the IRAS calibration.

The photometric standard star HR 8728 (Fomalhaut) was observed near in time and close in airmass to the observations of the HD stars. From its color-corrected IRAS flux densities, we calculate Fomalhaut's mid-infrared magnitude as $0.88 \mathrm{mag}$ from $8-18 \mu \mathrm{m}$ with an uncertainty of $10 \%$. On 21 Dec., observations of other standards at smaller airmasses are consistent with this magnitude and uncertainty for HR 8728 given typical Mauna Kea airmass corrections.

For HD 199143, the flux ratio of the binary was found by PSF fitting at $12 \mu \mathrm{m}$ to be $5.6 \pm 0.1$ and the total flux density from both components was measured in a 4". 8 synthetic aperture. The $18 \mu \mathrm{m}$ image had insufficient signal-to-noise ratio for the same PSF fitting procedure to work, so the flux densities were measured using small, diameter $1^{\prime \prime}$, aperture photometry around each star. The total flux density in a very large aperture agreed with the sum in the small ones. For HD 358253, the photometry of each component was found in a synthetic aperture of diameter 1" with residual background measured in an annulus 3". 5 from the source and subtracted. Statistical uncertainties calculated from the background were added in quadrature. The total uncertanties are reported in Table 4. Column two of this table represents the total integration time.
Table 4. Mid-infrared observations and results.

\begin{tabular}{lcccc}
\hline \hline $\begin{array}{l}\text { Filter } \\
\mu \mathrm{m}\end{array}$ & $\begin{array}{l}t_{\text {int }} \\
(\mathrm{s})\end{array}$ & Airmass & $\begin{array}{c}\text { primary } \\
(\mathrm{mJy})\end{array}$ & $\begin{array}{c}\text { secondary } \\
(\mathrm{mJy})\end{array}$ \\
& \multicolumn{5}{c}{ HD 199143 } \\
11.7 & 216 & 1.53 & $144 \pm 16$ & $32 \pm 4$ \\
17.65 & 240 & 1.55 & $66 \pm 25$ & $41 \pm 14$ \\
\multicolumn{5}{c}{ HD 358623 } \\
8.9 & 144 & 1.90 & $62 \pm 9$ & $34 \pm 7$ \\
11.7 & 240 & 2.2 & $35 \pm 5$ & $15 \pm 4$ \\
17.65 & 360 & 2.1 & $<20$ & $<20$ \\
\hline
\end{tabular}

\subsection{ROSAT and near-infrared imaging}

Voges et al. (2000) report a bright $(0.28 \mathrm{cts} / \mathrm{s}) \mathrm{X}$-ray source about one arc minute north of HD 199143 which itself is listed in the ROSAT All-Sky Survey. We examined ROSAT images of the field around HD 199143. With 30" pixelation, we see no indication at all of an X-ray source one minute north of the star. We also investigated the area north of the star with UCLA's twin-channel Gemini camera (McLean et al. 1994) at the Shane Telescope on 5 August 2001. A $60 \mathrm{~s} J$-band exposure with limiting magnitude $(3 \sigma)$ of 19.1 mag reveals no source $1^{\prime}$ to the north of HD 199143. There is also no indication on the corresponding ROSAT image of an X-ray source at the listed position.

\section{Results and discussion}

We use our photometry to place these stars in a colormagnitude diagram along isochrones by Baraffe et al. (2002) (Fig. 3). All four stars lie on or to the right of the $20 \mathrm{Myr}$ isochrone.

To investigate the reliability of Baraffe et al. isochrones at low masses, we selected $29 \mathrm{~K}$ and M-type dwarfs with less than $10 \%$ errors in parallax from the Gliese (1969) and Gliese \& Jahreiss (1991) catalogues. When added to the colormagnitude diagram (Fig. 3), these field stars, which we expect to be of main sequence age, parallel the Baraffe $100 \mathrm{Myr}$ isochrone. This is consistent with a pre-main-sequence phase for the HD 199143 and HD 358623 pairs.

Further evidence for the youth of HD 358623 comes from the $400 \mathrm{~m} \AA$ equivalent width of its $\mathrm{Li} \lambda 6708$ absorption line. Figure 1 of Song et al. (2002) - a plot of $(B-V)$ color vs. $\mathrm{Li} \lambda 6708 \mathrm{EW}$ - shows that all stars with equivalent widths $\sim 400 \mathrm{mÅ}$ belong to very young groups such as the TW Hydrae association, or the open cluster NGC 2264.

The ages of HD 199143 and HD 358623 are consistent with the $12_{-4}^{+8}$ age derived by Zuckerman et al. (2001) for members of the $\beta$ Pictoris Moving Group (BPMG) and confirmed by Ortega et al. (2002), who obtained a kinematic age of $11.5 \mathrm{Myr}$ by tracing the orbits of all proposed BPMG members backwards in time through a 3D Galactic potential. Furthermore, the Galactic space motion of HD 358623, relative to the Sun: $(-9.8 \pm 0.6,-15.5 \pm 0.3,-11.1 \pm 0.5)$, derived using Hipparcos distances and proper motions, is consistent with the mean motions $(-10.5,-15.8,-9.4)$ (Ortega et al. 2002) and velocity 


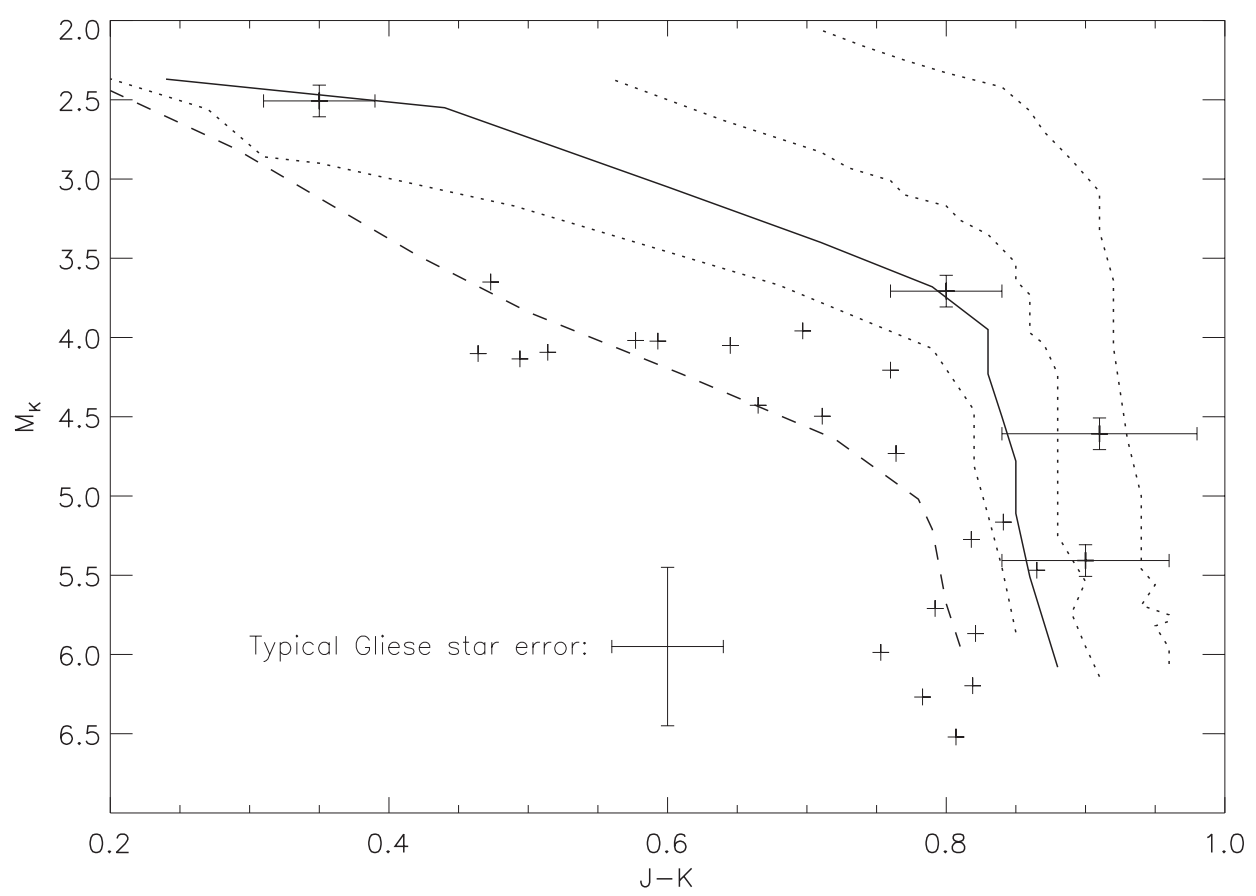

Fig. 3. Color-magnitude diagram showing (top to bottom) HD 199143 A, HD 358623 A, HD 199143 B, and HD 358623 B. Baraffe et al. (2002) isochrones, from left to right, are $100 \mathrm{Myr}$ (dashed line) $32 \mathrm{Myr}, 20 \mathrm{Myr}$ (solid line), $10 \mathrm{Myr}$, and 3 Myr. Positions of main-sequence Gliese stars are indicated by the "+" symbols. Colors are from the 2MASS catalogue.

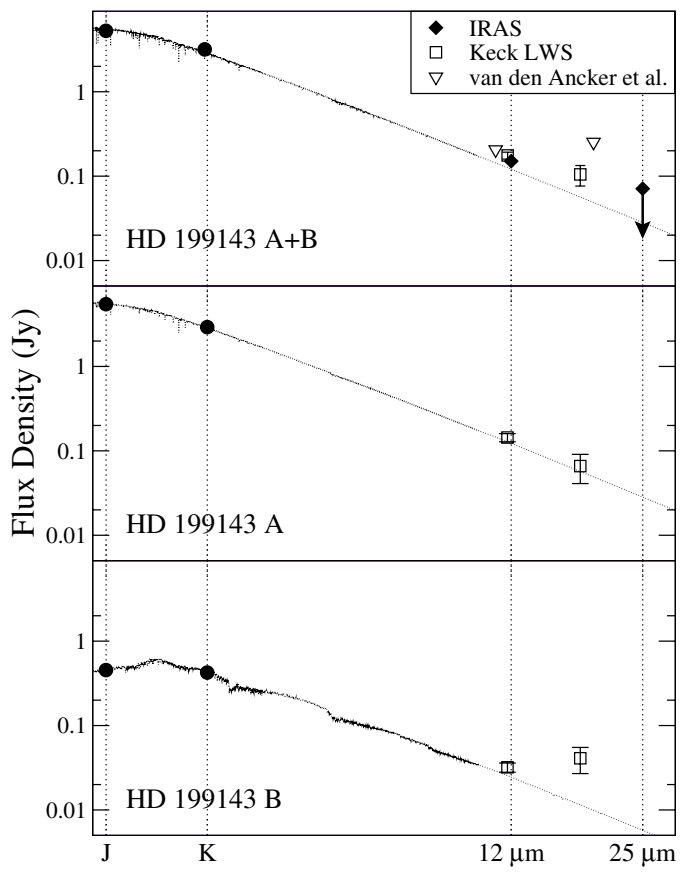

Fig. 4. Synthetic SEDs (Hauschildt et al. 1999) for HD 199143 A and $\mathrm{B}$ with overplotted mid-IR data. Effective temperatures for the models are $T_{\text {eff }}(\mathrm{A})=6400 \mathrm{~K}$ and $T_{\text {eff }}(\mathrm{B})=3700 \mathrm{~K}$, with $Z=0.02$ and $\log (g)=4.5$ for both models. Filled circles represent the photometric measurements of Section 2.1.

dispersions $\left( \pm \sim 2 \mathrm{~km} \mathrm{~s}^{-1}\right.$ in each direction) of the published BPMG stars (Zuckerman et al. 2001).

Figures 4 (HD 199143) and 5 (HD 358623) show synthetic spectra fit to the near-infrared photometry. In all four stars, the mid-infrared flux densities are consistent with those expected

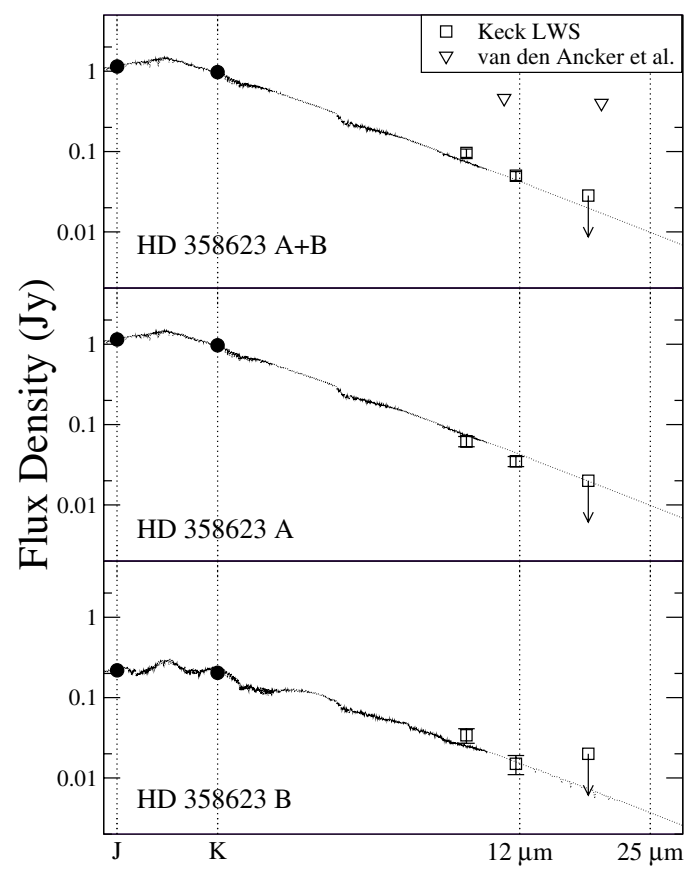

Fig. 5. Synthetic SEDs (Hauschildt et al. 1999) for HD 358623 A and $\mathrm{B}$ with overplotted mid-IR data. $T_{\text {eff }}(\mathrm{A})=4400 \mathrm{~K}$ and $T_{\text {eff }}(\mathrm{B})=$ $3300 \mathrm{~K}$, with $Z=0.02$ and $\log (g)=4.5$ for both models. The plotted upper limits at $17.65 \mu \mathrm{m}$ are 1 -sigma values. Filled circles represent the photometric measurements of Sect. 2.1.

from the photospheres. These results contradict the measurements of vdA00 and vdA01 by showing no substantial infrared excess. The small excess that appears around HD 199143B at $18 \mu \mathrm{m}$ is only significant at the $2 \sigma$ level. 


\section{Conclusions}

Data presented in this report show that HD 199143 AB and HD $358623 \mathrm{AB}$ are members of the $\beta$ Pictoris Moving Group, rather than a separate Capricornus group. The high lithium abundance of HD 358623 suggests an age of less than roughly $10 \mathrm{Myr}$ for this star. Near-IR adaptive optics photometry and models by Baraffe et al. (2002) also suggests a young age (less than $20 \mathrm{Myr}$ ). Our near- and mid-IR photometric results show no firm evidence for the presence of warm dust around either star. Investigation of a listed ROSAT All-Sky Survey source one arcminute north of HD 199143 revealed no near-IR counterpart to a limiting magnitude of $J=19.1 \mathrm{mag}$.

Acknowledgements. We would like to thank Randy Campbell, Jay Farihi, and Patrick Lowrance for their useful input to this study, as well as the referees, Drs. A. Brandeker and R. Liseau. This research was supported in part by a NASA grant to UCLA and by NASA's Astrobiology Institute. Some of the data presented herein were obtained at the W. M. Keck Observatory, which is operated as a scientific partnership among the California Institute of Technology, the University of California, and the National Aeronautics and Space administration. The Observatory was made possible by the generous financial support of the W. M. Keck foundation.

\section{References}

Baraffe, I., Chabrier, G., Allard, F., \& Hauschildt, P. H. 2002, A\&A, 382,563

Chauvin, G., Fusco, T., Lagrange, et al. 2002, A\&A, 394, 219

Diolaiti, E., Bendinelli, O., Bonaccini, et al. 2000, A\&AS, 147, 335
Elias, J. H., Frogel, J. A., Matthews, K., \& Neugebauer, G. 1982, AJ, 87,1029

Gliese, W. 1969 VeARI, 22, 1

Gliese, W., \& Jahreiss H. 1991, ARI Heidelberg, CD ROM

Hauschildt, P. H., Allard, F., \& Baron, E. 1999, ApJ, 512, 377

Jayawardhana, R., \& Brandeker, A. 2001, ApJ, 561, L111 (JB01)

Jones, B., \& Puetter, R. 1993, Proc. SPIE, 1946, 610

Lloyd, J. P., Liu, M. C., Macintosh, B. A., et al. 2000, Proc. SPIE, 4008, 814

Mathioudakis, M., Drake, J. J., Craig, N., et al. 1995, A\&A, 302, 422

McLean, I. S., Macintosh, B. A., Liu, T., et al. 1994, Proc. SPIE, 2198, 457

McLean, I. S. Beckin, E. E., Bendiksen, O.,et al. 1998, Proc. SPIE, 3354, 566

Neuhäuser, R., Guenther, E., Mugrauer, M., Ott, T., \& Eckart, A. 2002 A\&A, 395, 877

Ortega, V. G., Volkov, E., \& Monte-Lima, I. 2002, A\&A, 366, 276

Persson, S. E., Murphy, D. C., Krzeminski, W., Roth, M., \& Rieke, M. J. 1998, AJ, 116, 2475

Song, I., Bessell, M. S., \& Zuckerman, B. 2002 A\&A, 385, 862

van den Ancker, M. E., Perez, M. R., de Winter, D., \& McCollum, B. 2000, A\&A, 363, L25 (vdA00)

van den Ancker, M. E., Perez, M. R., \& de Winter, D. 2001, in Young Stars Near Earth: Progress and Prospects, ed. R. Jayawardhana, \& T. P. Greene (San Francisco: ASP), ASP Conf. Ser., 244, 69 (vdA01)

Voges, W., Aschenbach, B., Boller, Th., et al. 1999, A\&A, 349, 389

Voges, W. Aschenbach, B., Boller, Th. 2000, Max-Planck-Institut fur extraterrestrische Physik, Garching,

http://www . xray . mpe.mpg.de/rosat/survey/rass-fsc/

Vogt, S. S. 1987, PASP, 99, 1214

Zuckerman, B., Song, I., Bessell, M. S., \& Webb, R. A. 2001, ApJ, 562, L87 